\title{
The Difficulty and Significance of NursingPracticeUsing Complementary and Alternative Medicine (CAM)
}

\author{
Mas ako Nagase*, Mayumi Takaya \\ Juntendo University, Faculty of Health Care and Nursing
}

\begin{abstract}
Japan's national healthcare system is based solely on modern medicine. This study aims to clarify the expectations surrounding CAM and the factors hindering the practice of CAM in nursing. The in formants for the interview survey were five nurses who have had professional training in aro matherapy. We asked them about their expectations ofCAM and about the difficulty of nursing care as a CAM practitioner. Whether or not informants were using CAM in their work, they understood the difficulty nursesface in attempting to practice CAM therapy.We found thatapproval of the boss and co-workers is indispensable for using CAMin nursing care. In addition, the practitioner must show evidence that the therapy works. Informants spoke of the difficultyofshowing evidence when it is not possible to do a clinical study. On the other hand, the view on the value of CAMnursing may be revisedif nurses can show the evidence for its efficacy and if the therapy can become more widespread.Nursing science may be able to contribute to the evidence, and healthcare system reform could facilitate its wider use.
\end{abstract}

Keywords Nursing Care, Comp lementary and Alternative Medicine, Feasibility, Aro matherapy, Ly mphatic Drainage

\section{Introduction}

Over the past decade, there has been a growing interest in complementary and alternative medicine (CAM) in nursing[1][2]. As of 2009, 581 nursing articles about CAM had been published in the Japan CentraRevuoMedicina, which accounted for $5.4 \%$ of all articles $(14,483)$, including both medicine and psychology[1]. Although the proportion of nursing articles is still very low, the number of nursing articles increased 5 times from 1998 to 2007, suggesting an increasing awareness of CAM in nursing practice.

According to Snyder, nurses provide holistic care, which could include CAM[3]. In Japan, however, such practices as aromatherapy and reflexology, which are part of CAM, are not considered to be a part of nursing care.Many nurses, however, consider that it is important to adopt CAM in clinical nursing[4][5][6], and many of them actively participate in seminars to study CAM[5][6].

In our survey[7], which involved nurses working at university hospitals in the Tokyo Metropolitan Area, the results indicated a growing awareness of CAM, especially aromatherapy, reflexology, and music therapy. However, of the $92.7 \%$ of nurses who considered it important to adopt CAM in nursing, those who have actually administeredit accounted for only $52.1 \%$.

* Corresponding author:

mnagase@juntendo.ac.jp (Masako Nagase)

Published online at http://journal.sapub.org/sociology

Copyright (C) 2012 Scientific \& Academic Publishing. All Rights Reserved
One of the reasons for the relative scarcity of CAM practice may be that an appropriate social network is lacking[8]. A social network fulfils the function of providing social mechanisms that affect individual behavior and thoughts, and by which people determine their social actions; that is, action are influenced less by personal qualities, such as interests and abilities, and more by other members of the social network - in the context of nursing, then, by other nurses.Our report[7] also showed an association between the use of CAM and social networks;nurses might receive support from friends or colleagues who share their interest in CAM. On the other hand, we found no association between an interest in CAM and its practice, suggesting that the determination to perform CAM is more closely affected by relationships with colleagues rather than by personal factors, such as an interest in or learning of CAM.

Another possible reason is that the efficacy of CAM has not been "scientifically" proven, which has led people either to have negative views about CAM or to have suspended judgment[9][10][11]. In fact, clinical nurses consider that professional knowledge and skills as well as scientific evidence are essential in order to use CAM in nursing practice[7].

As Snyder pointed out, if nursesare supposed to function as "holistic" nursing practitioners, it is inevitable that they will have an interest in and expectations for CAM interventions. Despite the many problems that exist in using CAM in nursing practice, it may be necessary to create an environment where CAM can be practiced if it is considered a necessary nursing intervention. Thus, this study searched 
for causes of the difficulties in using CAM in nursing practice.We administered an interview survey involving nurses who try to acquire professional knowledge of and skills with aromatherapy or ly mphatic drainage for regular use in clin ical settings.

\section{Purpose}

The purpose of this paper is toclarify the difficulty of using CAM therapy in routinenursing practice. In addition, wediscuss the significance of CAM therapy use and what it means for nursesto lettheir bosses and colleaguesknow that they use CAM therapy.

$<$ De fin itions $>$

Complementary and alternative medicine: Aromatherapy, lymph drainage and music therapy; general terms for treatments complementing or substituting conventional med icine.

Therapy: Refers to individual therapies that form a part of CAM.

\section{Method}

\subsection{Selection of Target Population}

In order to participate in the survey, informants had to (a) be registered nurses, (b) have learned about at least one CAM therapy, such as aromatherapy or ly mph drainage, and have the knowledge and techniques to practice it, regardless of formal qualification; and (c) use the therapy routinely or intend to use it.

\subsection{Ethical Consider ations}

Before the interview, we explained to informants the purpose of the study, the methods, and ethical considerations, after which they gave written consent. A withdrawal form was also distributed in advance for informants who might wish to leave the study after the interview. This study was approved by the Ethics Committee for the Faculty of Health Care and Nursing,Juntendo University.

\subsection{Data Collection}

Method: Semi-structured interviews were conducted, during which informants' statements were recorded with an IC recorder and transcribed later. Each interview lasted about 60 minutes. The interview questions, which were divided into two groups, are as follows:

a. Personal experience of therapy effectiveness

When did you get an opportunity to study these therapies?

Why do you think these therapies should be introduced into nursing care?

b. Howhave nursing care treatments been structured;how should they be structured?

How do colleagues or other workers feel?

Which areas do you think have been well implemented?

Which areas do you think are not well implemented?

\subsection{Analysis}

Our method was analytic induction.We classified the interview content based on the following factors: feasibility of implementation, results obtained from trial practice, and opportunities for introducing this therapy into nursing care.Then, we coded each person's response. We then categorized it to detect any similarity between the codes.

\section{Results}

The nurses who participated in this study are described as $\mathrm{A}, \mathrm{B}, \mathrm{C}, \mathrm{D}$, and $\mathrm{E}$. The categories and subcategories are shown in [] and $\diamond$.

\subsection{General Over viewof the Infor mants}

The informants were five female nurses, consisting oftwo nurses who have job titles (chief nurse and head nurse) and three staff nurses. Only DusesCAM in routine work.Of the five nurses, only $\mathrm{D}$ has been regularly providing complementary therapies (Table 1).

Table 1. Informant summary

\begin{tabular}{|c|c|c|c|c|c|}
\hline Informants & $\mathrm{A}$ & $\mathrm{B}$ & $\mathrm{C}$ & $\mathrm{D}$ & $\mathrm{E}$ \\
\hline Gender & Female & Female & Female & Female & Female \\
\hline \multirow{2}{*}{ Therapy practiced } & \multirow[t]{2}{*}{ Aromatherapy } & \multirow[t]{2}{*}{ Aromatherapy } & \multirow[t]{2}{*}{ Aromatherapy } & Aromatherapy & \\
\hline & & & & Lymph drainage & Lymph drainage \\
\hline Qualification & $\circ$ & $\circ$ & $\circ$ & $\circ$ & - \\
\hline Cont inued practice & - & - & - & $\circ$ & - \\
\hline Duty position & Staff nurse & Staff nurse & Staff nurse & Head nurse & Chief nurse \\
\hline The assigned post & Hospital ward & Hospital ward & Hospital ward & $\begin{array}{c}\text { Nursing } \\
\text { management } \\
\text { section } \\
\end{array}$ & Hospital ward \\
\hline Nursing hist ory & $8-10$ years & $5-7$ years & 5-7 years & $30-35$ years & $20-25$ years \\
\hline $\begin{array}{l}\text { Years of service in the } \\
\text { currently held position }\end{array}$ & About 3 years & About 3 years & About 3 years & Over 5 years & About 3 years \\
\hline
\end{tabular}




\subsection{Motivation for Learning CAM Therapy}

The nurseswere motivated by their own [personal experience], which drove them to acquire the professional therapeutic knowledge, skills, and certification to practice in clinical settings. Table 2 shows the elements included in [personal experience].

Table 2. Opportunities

\begin{tabular}{c}
\hline [Personal Experience $]$ \\
Personal interest \\
Identity as a Nurse \\
Encounters with patients \\
\hline
\end{tabular}

Informant A stated that she has been interested in scents since childhood. She knew that scents were used in a treatment called aromatherapy before starting to work as a nurse. Her <personal interest> in scents facilitated an opportunity to purchase the essential oils used in aromatherapy when she was suffering from insomnia after starting the job. The oils worked to help alleviate her insomnia, which led her to explore ways of adopting complementary therapy in nursing practice.

Informant $\mathrm{D}$ also had an experience of healing which led to her own $<$ personal interest $>$.

I had a personal interest in aromas and wondered if they could be used for cure or relaxation...Therefore, I performed some experiments and believed that they could probably be used in nursing care. (D)

Informant $C$ was searching for her $<$ identity as a nurse $>$ and asking herself how she wanted to be involved in nursing care, what kind of care she wanted to provide, and what kind of nurse she wanted to be. These questions motivated her to explore the possibilities of using her skills in nursing:

It was just around autumn of my 3rd year when I began to question why I had become a nurse and what I really wanted to do. I began to wonder what type of nurse I wanted to be as I con tinued my position in the ICU. I looked back and when I thought about what I wanted to do, aromatherapy came to mind. (C)

At least two of the nurses became motivated to adopt CAM through an <encounter with patients>. Informant E described her feelings when she could not do anything for patients with lymphedema, and informant $\mathrm{B}$ recounted her experience of providing aromatherapy for patients during practical training when she was a nursing student, which facilitated smooth communication with the patients.

\subsection{Causes of Difficultiesin Regular Use ofCAM}

Factors that encourage the use of complementary therapy in nursing practice were determined to be [professional support], [educational opportunity], and [establishment of a system] (Table 4).

Regarding [professional support], informants stated that approval and support from a head nurse and nurses holding administrative positions greatly affected the implementation of complementary therapy in clinical settings, indicating the need of <understanding and support from the boss>.
Informants $\mathrm{C}$ and $\mathrm{D}$ related their experiences concerning support:

At the time, our head nurse said that he or she would love to cooperate, which was a great help. The nurse came to this ward because he or she wanted to practice aromatherapy...I thought about what I could do, and I wanted to take care of $C$ and $X$, who were transferred here... They said that they wanted to convey the message that aromatherapy can be safely used in nursing care and that it was suitable for other staff to use in nursing care. (C)

Regarding massage I provide for patients using essential oils, a physician once laughed at me, saying, "Is this a part of nursing care?" or "Is this something a nurse has to do?" At that time, our nurse manager stood up for me...(snip)...There may be many problems to overcome for a young nurse to adopt complementary therapy, but I think it is possible, by obtaining theunderstanding of a head nurse, to conduct it as a part of the hospital service, or negotiating with other physicians. (D)

Table 4. Factors making treatment possible

\begin{tabular}{c} 
[Professional support] \\
Understanding and cooperation from superiors \\
Understanding and cooperation from colleagues \\
\hline [Educational opportunity] \\
Knowledge and skills to guarantee quality of care \\
Support from nursing schools \\
\hline [Establishment of a system] \\
Understanding from other professions \\
Difficulty in showing evidence \\
System structure
\end{tabular}

Thus, in both cases, support from the head nurse encouraged these nurses, which led to an implementation of complementary therapy in nursing practice.

Informant B talked about an incident caused by the use of essential oils, which led to a trend in which nurses refrained from practicing aromatherapy, and their studies on complementary therapy were suspended by the adminis trative division of nursing.

In one of our hospital wards, aromatherapy massage was given to a patient for whom the use of essential oils was contraindicated, and it aggravated his/her inflammatory skin condition. This incident gave a bad impression of aromatherapy to other nurses, which made them think [that] aromatherapy is not good. (B)

Informant A described her experience of continuing aromatherapy with <understanding and support from colleagues $>$ in the absence of $<$ understanding and support from the head nurse $>$.

I was told by a head nurse who had just taken up a position here that "we will not practice aromatherapy." However, there was an underlying sentiment among the staff who had been working here for a while that we [had] always practiced aromatherapy here, and the patien ts wanted it. Therefore, we would have liked to keep it that way. So, we continued on our own, without persuading the head nurse. (A)

In terms of [educationalopportunity], the $<$ knowledge and skills to guaranteethe quality of care $>$ can be acquired without certification; however, the care should be provided with the cooperation of a person whois professionally 
qualified or with a nurse who has professional knowledge and skills. $<$ Support from nursing schools $>$ is necessary for helping to provide opportunitiesfor learning and exploring new care techniques.

Regarding the [establishment of a system], the informants pointed out the importance of several factors.Concerning <understanding from different types of health care professionals $>$, physicians' lack of understanding about nursing care makes it difficult to practice complementary therapy, as shown by the experience of informant $D$ regarding <understanding and support from the boss $>$. The informants stated that "[p]hysicians do not disagree with conducting complementary therapy as nursing practice" (B and C) and "[p]hysicians kindly offered to cooperate with data collection when we conducted complementary therapy as a part of research" (C), suggesting that physicians do have cooperative and positive attitudes towards therapy. However, physicians tend to be skeptical about the pharmacological effects of the therapy, and do not agree with the use of therapy as complementary medicine (B, C, and D). Informant $\mathrm{D}$ also said, "Aromatherapy or massage was expected to complement the effects of physiotherapy." Informant D went on to criticize a physiotherapist who regarded nursing practice as complementary to physiotherapy, despite the fact that the functions of nursing and physiotherapy practice are supposed to be separate, and suggesting that informant $\mathrm{D}$ was trying to insist that the use of complementary therapy is an autonomous nursing practice.

To overcome < difficulties in showing evidence>, it was considered important to conduct many studies to accumulate scientific evidence for the therapy in order to obtain <understanding and support from the boss $>$ and $<$ understanding and support from colleagues $>$. However, to conduct a study, <understanding and support from the boss $>$ was considered essential ( $B$ and $C$ ).

To pro mote $<$ a system structure $>$, in formants considered it important to prepare a guideline that could ensure clinical safety and to allocate necessary resources so as not to affect other operations. They also considered it essential to secure time for nurses to administer aromatherapy and lymphatic drainage, as well as specify that these therapies are a part of nursing practice (A, B, C, and D).

\subsection{Feelings Regarding Treatment Efficacy}

Table 3 shows the effects of therapy identified from participants' responses.

Table 3. Feelings regarding treatment efficacy

\begin{tabular}{c}
\hline [Symptomatic relief] \\
Alleviation of physical distress \\
Reduce swelling \\
Improvement of hormonal balance \\
\hline Psychological effects] \\
Relaxation \\
Nervous system stability \\
\hline [Social effects] \\
Facilitation of connectedness \\
Feeling of familial satisfaction
\end{tabular}

Regarding [symptomatic relief], informants A, B, and C commented on the <alleviation of physical distress>, in which aromatherapy may not take the symptoms away, but does help to relieve the m temporarily, noting also that it will not help if the patient doesn't like it.

For example, I don't use lavender just because it is supposed to help with relaxation. If I want a patient to relax and they don't like lavender, it won't help them relax at all. (D)

To ease pain and malaise, aromatherapy can only help a little with the symptoms that the patients are concerned about; for the rest, I think it is better if they can spend some quality time with their families. (A)

Aromatherapy is also effective to <promote hormonal balance>, especially in wo men.

Informant D spoke about the effects of lymphatic drainage by comparing them with those of aromatherapy:

Since lymph drainage treatment is highly effective, patients are happy too. With intensive treatment over several days, even if only once a week, results are observed within a few weeks. Patients can wear shoes that they could not fit into earlier, wear wedding or funeral clothes that now fit properly, and start leading a normal life. You can see the results of lymph drainage. (D)

Thus, the purpose of ly mphatic dra inage treatment, which is to $<$ reduce swelling $>$, is specific, and its effects are clearly visible.

Regarding [psychological effects], the above-mentioned $<$ alleviation of physical distress $>$ is usually generated as a result of $<$ relaxation $>$.

Like people say: "Illness starts in the mind" ...Patients undergoing chemotherapy often experience severe nausea, [which is] affected by their mental state. When I used aromatherapy for a patient who always complained of nausea, he or she could feel a little relaxed, and the side effects of chemotherapy were reduced ... (B)

In stating that <alleviation of physical distress $>$ was achieved by producing $<$ relaxation $>$, she pointed out that aromatherapy does not necessarily have pharmacological effects.

As a therapist, I have learned that it is illegal to mention pharmacological effects to patients, such as a blood pressure-lowering effect, diuretic effect, or pain-relieving effect. To tell someone about the effects of aromatherapy as a nurse, I should say [that] it can aid relaxation, promote restful sleep, and reduce anxiety... (B)

Aroma and gentle touching gave patients a "sense of being cared [for]," which is considered to contribute to creating a restful mo ment in which patients can attain < peace of mind $>$.

By having such a relaxing time, I felt that it promoted smooth communication and helped establish a closer bond with the patients. So aromatherapy can be used as a tool for enhancing communication. (D)

Concerning the [social effects], in formants A, B, and C mentioned the $<$ facilitation of connectedness $>$ because the use of aro matherapy provided them with an opportunity to deepen relationships with the patients. In terms of the $<$ feeling of familial satis faction $>$, informants A and D stated that enriched nursing care can prevent a patient's family 
from developing loneliness and can facilitate a sense of satisfaction.

For terminal patients, creating their own private space was possible ... I could feel that their families were also fully satisfied with the care we provided ...(C)

\section{Discussion}

In this study, the informants were motivated by their [personal experience], which drove them to acquire professional knowledge of CAM to practice in clinical settings. However, no systematic and strategic approach was observed in the process of initiating complementary therapy. This may be one of the factors hampering the adoption of CAM.

The first reason that nurses have difficulty in adoptinga systematic and strategic approach may be that the direct medical care provided must be based on physicians' competence. CAM therapy sometimes infringes on a physician's practice, depending on its purpose and the types of therapy. There fore, in order to integrate CAM into nursing practice, it is essential to show strategically that complementary therapies are a part of nursing practice that does not infringe on areas of a physician's practice.

The informants in this study saw or experienced [symptomatic relief], [psychological effects], and [social effects] by means of complementary therapy. However, they also stated that [symptomatic relief] is generated only by [psychological effects] and [social effects]. Co mplementary autonomy between nurses and physicians was maintained by strategically confining the purpose of nursing practice to providing holistic care to patients. When roles and tasks overlapped among multiple health care providers, such strategic confinement was used to maintain relationships so that they could respect each other, implement their own tasks, and cooperate with other workers[12]. However, if complementary therapy does not threaten to infringe on other clinical areas of practice, a systematic and strategic approach may not be necessary for adopting CAM.

The second reason is that nursing care involves "social action." Within a structure, the concepts or interests of people who have admin istrative positions often influence the organizational aims. Informant A stated that the implementation of complementary therapy is possible if staff nurses assume that the use of aromatherapy is to achieve [symptomatic relief] or [psychological effects], even if $<$ understanding and support from the boss $>$ are not obtained. However, this is only possible when staff members have the same purpose-to share in a structurally stable network-and if <understanding and support from colleagues $>$ are obtained.

The third reason is that the effectiveness of CAM has not been clinically proven[5][6][10][11]. Currently, awa reness of CAM is increasing among nurses[1][9], and social awareness is also growing in terms of health promotion and maintenance[11]. However, it is difficult to adopt treatment without evidence as part of professional nursing care.
The fourth reason is that even if professional and special nursing care services are provided, most complementary therapies are not included in the cost of medical care in the Japanese medical system. Complementary therapies, such as aromatherapy, are costly and time-consuming, but the current Japanese medical insurance system cannot charge fees for its service. Lymphatic drainage is recognized as part of lymphatic edema treatments, so its medical fees were specified under the medical insurance system in 2008. Despite the fact that it has limitations in terms of the types of disease and levels of conditions, the insurance system promoted the implementation of the therapy.

These four aspects are not considered specific to the implementation of CAM in nursing practice. Because nurses are health care professionals, they are supposed to have the knowledge and skills specific to nurses and to control their autonomous practices at their own discretion. In reality, however, they face many of the problems discussed above. In Japan, a law regarding educational programs for nurturing advanced practice nurses will be implemented after April 2012. Fundamental systemic reforms are essential for empowering nurses to provide care for patients. However, even in the process of discussion about nurturing advanced practice nurses, the government still maintains a policy of protecting and keeping conventional areas of physicians' practice, preventingnurses' use of discretionary power, and maintaining an extremely conservative stance. Therefore, even if nurses wish to provide better care for patients and attempt strategic confinement regarding their practice, the adoption of CAM may not be possible without dynamic systemic reforms.

\section{Conclusions}

The consent of the boss and cooperation of colleaguesare necessary for a nurse to use CAM as a common practice. In part, such cooperation is necessaryfor using CAM because of the time it takes;therefore, a particular time must be foundfor using CAM therapy. Study participants felt that CAM would need to be institutionalized so that it could be made a part of a nurse's regular duties.

Nurses' use of CAM suggests the possibility of a medical change, though, and the science of nursing is beginning to promote it. Perhaps the evidence that is neededfor using CAM therapy safely will be produced through further studies.

\section{ACKNOWLEDGEMENTS}

We would like to thank the nurses who participated in this study. This study was carried outin 2010-2011 with the aid of the Collaborative Research Fund of the Faculty of Nursing and Medical Care. 


\section{REFERENCES}

[1] Nagase, M., \& Takaya, M. et al., Status of complementary and alternative therapies for nursing research.30th Proceedings Annual Meeting of the Japanese Scientific Nursing Association, 508, 2010.

[2] Nakamura, N., 2008 The present condition and its market size of the alternative medicine of our country. Studies in social sciences, 124, 69-91, 2008.

[3] Snyder, M. \&Lindquist, R., Complementary\&Alternative Therapies in Nursing $3^{\text {rd }}$ edition. New York: Springer Publishing Company, 1998.

[4] Narui, H., \& Suita, Y., et al., Nurses' attitudes toward alternative cancer therapies.Journal of Aomori University of Health and Welfare, 7(2), 177-186, 2006.

[5] Nitta, K. \& Kawabata, K., Current Situation and Issues of Complementary and Alternative Medicine in Nursing.Journal of Japanese Complementary and Alternative Medicine, 41(1), 23-31, 2007.

[6] Takaya, M., \& Nakajima, Y., et al., Studies on the use of alternative therapies in nursing: Survey of clinical nurse, Journal of Health Care and Nursing, 6(1), 113, 2010.
[7] Nagase, M., \& Takaya, M., et al., Interest in complementary and alternative medicine (CAM) among nursing professionals and issues in using CAM as part of nursing care: Questionnaire survey of nurses working in the Tokyo metropolitan area. Journal of Health Care and Nursing, 7(1), 41-46, 2011.

[8] Yasuda, Y.,Network Analysis: How do you determine what actions. Tokyo: Shiny osya, 1997.

[9] Singh, S. \& Ernst, E.,Trick or Treatment? Alternative Medicine on Trial, London: Corgi, 2009.

[10] Weiger, W. Q. et al.,Advising patients who seek complementary and alternative medical therapies for cancer. Annals of Internal Medicine, 137, 889-913, 2002.

[11] Sato, J., Medicine. In K, Kuroda (Eds.), Modern medical sociology: Japan's current situation and challenges (pp. 2-32). Kyoto: Sekaisisosy a, 1999.

[12] Mitsui, S.,Sociology of care: Dialogue with the clinical.Tokyo: Keisosyobo.2004. 